\title{
Type 1 Idiopathic Macular Telangiectasia Associated with Type 3 Neovascularization
}

\author{
Julien Tilleul Giuseppe Querques Vittorio Capuano Alexandra Miere \\ Mayer Srour Eric H. Souied
}

Department of Ophthalmology, Centre Hospitalier Intercommunal de Créteil, University Paris Est Créteil, Créteil, France

\section{Key Words}

Anti-vascular endothelial growth factor · Fluorescein angiography · Idiopathic macular telangiectasia Spectral-domain optical coherence tomography - Type 3 neovascularization

\begin{abstract}
Purpose: To report the case of a patient with unilateral idiopathic macular telangiectasia (IMT) associated with type 3 neovascularization. Methods: Observational case report. Results: We describe a case of an 85-year-old woman who presented at our department with a gradual vision loss in her left eye (LE). Her best-corrected visual acuity (BCVA) was 20/200 in the LE. Fundus examination showed 2 small hemorrhages located nasally to the LE fovea, as well as lipid exudates. Fluorescein angiography revealed early hyperfluorescence corresponding to the dilated capillaries. Spectral-domain optical coherence tomography (SD$\mathrm{OCT}$ ) showed several microaneurysms within the inner retinal layers. Late indocyanine green angiography revealed a focal hyperfluorescence corresponding to a type 3 neovascularization. No signs of IMT or type 3 neovascularization were detected in the right eye. Based on these findings, the patient was diagnosed with type 1 IMT and coincident type 3 neovascularization. The LE was treated with intravitreal anti-vascular endothelial growth factor (antiVEGF) injections. Twenty-four months later, SD-OCT revealed regression of the exudative signs, and LE BCVA improved to 20/100. Conclusion: We describe the case of an unusual association between older-onset IMT and type 3 neovascularization, and subsequent regression by anti-VEGF injections. We propose a new IMT subtype called type $1 \mathrm{C}$ for this association. Further research must be done in order to establish the pathophysiologic mechanism and likelihood of this association.

(c) 2014 S. Karger AG, Basel
\end{abstract}


Tilleul et al.: Type 1 Idiopathic Macular Telangiectasia Associated with Type 3 Neovascularization

\section{Introduction}

In 1982, Gass and Oyakawa [1] described idiopathic macular telangiectasia (IMT) as a condition involving capillary ectasia and dilation in the juxtafoveal region. Clinical presentation comprises decreased visual acuity, sometimes accompanied by metamorphopsia, as well as a central scotoma, as the disease slowly progresses.

According to the classification by Gass and Blodi [2], there are 3 types of IMT (table 1). Type 1 (IMT-1): unilateral macular telangiectasia with visible aneurysms, which occurs predominantly in males; in this case, the cause of visual loss is mostly macular edema [visible and exudative IMT (IMT-1A); visible, exudative and focal IMT (IMT-1B)]. Type 2 (IMT-2): bilateral macular telangiectasia that occurs in men and women, with characteristic features on multimodal imaging [3]; subretinal or intraretinal neovascularization is frequently seen in this type [occult and nonexudative IMT (IMT-2A); juvenile, occult and familial IMT (IMT-2B)]. Type 3 (IMT-3): very rare, associated with vascular occlusions, occurring in the larger setting of a medical or neurological disease [occlusive IMT (IMT-3A); occlusive IMT associated with central nervous system vasculopathy (IMT-3B)].

Moreover, in 2006, Yannuzzi et al. [4] merged the IMT-1 subtypes into one group of aneurysmal telangiectasia, characterized by clearly visible aneurysms, and defined IMT-2A as a group of perifoveal telangiectasia, leaving out IMT-2B and IMT-3 because of their rarity. In addition, they described the possible association of IMT- 2 with retino-retinal anastomosis and choroidal neovascularization [4].

The therapeutic options for IMT-1 consist of laser photocoagulation, which is the main treatment, as well as intravitreal injections (IVTs) of steroids or anti-vascular endothelial growth factor (anti-VEGF) agents [3].

Several associations have been found between the IMT and other vascular anomalies [5]. The case we report combines the characteristics of IMT-1A with the intraretinal vascular complex typical of a type 3 neovascularization.

\section{Case Report}

An 85-year-old woman presented at our clinic complaining of gradual vision loss in her left eye (LE). Her past medical history included well-controlled arterial hypertension. Her best-corrected visual acuity (BCVA) was 20/80 in the right eye (RE) and 20/200 in the LE. The anterior segment examination was unremarkable. Fundus examination of the RE was unremarkable, while for the LE, it revealed 2 small hemorrhages located nasally to the fovea accompanied by lipid exudates inferiorly and superiorly (fig. 1).

Early-phase fluorescein angiography (FA; Spectralis, SD-OCT+HRA, Heidelberg Engineering, Germany) was unremarkable in the RE, while it demonstrated dilated capillaries within the LE fovea, along with vascular tortuosity in the superior and nasal macula (fig. 2a). Early FA of the LE also showed a zone of focal hyperfluorescence with a superotemporal right-angle dilated feeding vessel, deepening into the foveal avascular zone, evocative of a type 3 intraretinal neovascularization (fig. 2a). Spectral-domain optical coherence tomography (SD-OCT; Spectralis) of the RE showed an epiretinal membrane along with a discrete vitreomacular traction. SD-OCT of the LE revealed small, round, well-demarcated foveal lesions with hyperreflective edges in the inner retinal layers, corresponding to microaneurysms along with cystoid spaces. A hyperreflective lesion located in the outer retinal layers, temporal to the macula and adherent to the retinal pigment epithelium [6, 7], indicating the presence of type 3 intraretinal neovascularization, was also noted (fig. 2b). Indocyanine 
Tilleul et al.: Type 1 Idiopathic Macular Telangiectasia Associated with Type 3 Neovascularization

green angiography (ICGA; Spectralis) revealed a late hyperfluorescence and confirmed the presence of a type 3 neovascularization in the LE (fig. 2c).

Based on these findings, the patient was diagnosed with atypical IMT-1 (the average age at presentation of patients in this group is 37 years [2]) and coincident type 3 neovascularization. The patient was treated with a series of 3 monthly IVTs of ranibizumab $(0.5 \mathrm{mg} / 0.05$ $\mathrm{ml}$ ) in the LE followed by a prn regimen.

At 6 months, after 3 ranibizumab IVTs, BCVA improved to 20/160 in the LE. FA and ICGA showed absence of late-phase leakage from the lesion, and SD-OCT revealed regression of the exudative signs (fig. 2d). SD-OCT revealed recurrence of exudative signs at 8 months, and thus, the patient received repeated ranibizumab IVTs on a monthly basis up to month 14 (6 IVTs). At month 16, due to recurrence of exudative signs, the patient received repeated ranibizumab IVTs on a monthly basis up to month 22 (6 IVTs). At 24 months, after a total of 15 IVTs, BCVA improved to 20/100 in the LE, and FA, ICGA and SD-OCT showed regression of both cystoid macular edema and serous retinal detachment.

\section{Discussion}

We described the case of a patient diagnosed with atypical IMT-1A associated with type 3 neovascularization. According to the classification by Gass and Blodi [2], the presence of a unilateral telangiectatic lesion accompanied by microaneurysms would suggest the diagnosis of IMT-1A. Nevertheless, the association of macular telangiectasia with a type 3 intraretinal neovascularization is a typical complication of IMT-2 and is known to be a sign of exudative age-related macular degeneration [8, 9]. To our knowledge, the shared characteristics between IMT-1 and IMT-2 have only been described once in a similar case in 2011, by Mezad-Koursh et al. [10]. Given the unusual association observed, our cases, along with the case described by Mezad-Koursh et al. [10], are evocative of a new subtype of IMT that we propose to call IMT-1C, characterized by unilateral macular telangiectasia with visible aneurysms and macular edema, possibly complicated by type 3 neovascularization. This subtype of IMT seems responsive to anti-VEGF treatments. The description of more cases of IMT-1C is desirable in order to define this subtype of IMT.

\section{Disclosure Statement}

The authors have no proprietary interest in the materials used in this study and received no financial or material support for the research and the work.

\section{References}

1 Gass JD, Oyakawa RT: Idiopathic juxtafoveolar retinal telangiectasis. Arch Ophthalmol 1982;10:769-780.

2 Gass JD, Blodi BA: Idiopathic juxtafoveolar retinal telangiectasis. Update of classification and follow-up study. Ophthalmology 1993;100:1536-1546.

-3 Nowilaty SR, Al-Shamsi HN, Al-Khars W: Idiopathic juxtafoveolar retinal telangiectasis: a current review. Middle East Afr J Ophthalmol 2010;17:224-241.

-4 Yannuzzi LA, Bardal AM, Freund KB, Chen KJ, Eandi CM, Blodi B: Idiopathic macular telangiectasia. Arch Ophthalmol 2006;126:450-460.

5 Atmani K, Querques G, Zourdani A, Benhamou N, Souied EH: Unusual presentations of type 2 idiopathic macular telangiectasia. Ophthalmologica 2013;230:126-130. 
Tilleul et al.: Type 1 Idiopathic Macular Telangiectasia Associated with Type 3 Neovascularization

-6 Querques G, Souied EH, Freund KB: Multimodal imaging of early stage 1 type 3 neovascularization with simultaneous eye-tracked spectral-domain optical coherence tomography and high-speed real-time angiography. Retina 2013;33:1881-1887.

7 Querques G, Atmani K, Berboucha E, Martinelli D, Coscas G, Soubrane G, Souied EH: Angiographic analysis of retinal-choroidal anastomosis by confocal scanning laser ophthalmoscopy technology and corresponding (eye-tracked) spectral-domain optical coherence tomography. Retina 2010;30:222-234.

8 Engelbert M, Yannuzzi LA: Idiopathic macular teleangiectasia type 2: the progressive vasculopathy. Eur J Ophtalmol 2013;23:1-6.

-9 Slakter JS, Yannuzzi LA, Schneider U, Sorenson JA, Ciardella A, Guyer DR, Spaide RF, Freund KB, Orlock DA: Retinal choroidal anastomoses and occult choroidal neovascularization in age-related macular degeneration. Ophthalmology 2000;107:742-753, discussion 753-754.

-10 Mezad-Koursh D, Zayit-Soudry S, Barak A, Loewenstein A: Unilateral idiopathic macular telangiectasia with choroidal neovascularization. Ophthalmic Surg Lasers Imaging 2011;42:E1-E3.

Table 1. Epidemiological and clinical features of IMT, according to the classification by Gass and Blodi [2]

\begin{tabular}{|c|c|c|c|c|c|c|}
\hline & \multicolumn{6}{|l|}{ IMT type } \\
\hline & $1 \mathrm{~A}$ & $1 \mathrm{~B}$ & $2 \mathrm{~A}$ & $2 \mathrm{~B}$ & $3 \mathrm{~A}$ & $3 \mathrm{~B}$ \\
\hline Frequency & $\begin{array}{l}\text { Second most } \\
\text { common }\end{array}$ & Rare & Most common & Extremely rare & Very rare & Very rare \\
\hline Gender & Male (90\%) & Male & Male $=$ female & Male $=$ female & Female & Male $=$ female \\
\hline Age, years & $15-54$ & Middle age & $35-65$ & $35-65$ & $40-60$ & Middle age \\
\hline Congenital/acquired & Congenital & Congenital & Acquired & Acquired & Acquired & $(?)$ \\
\hline Laterality & Unilateral & Unilateral & Bilateral & Bilateral & Bilateral & Bilateral \\
\hline Microaneurysms or telangiectasia & +++ & + & - & - & + & + \\
\hline Yellow exudates & + & $+/-$ & - & - & Minimal & Minimal \\
\hline Subretinal neovascularization & - & - & + & + & - & - \\
\hline Capillary occlusion & Minimal & - & - & (?) & +++ & +++ \\
\hline Systemic associations & - & - & - & - & - & $\begin{array}{l}\text { Central nervous } \\
\text { system } \\
\text { vasculopathy (?) }\end{array}$ \\
\hline
\end{tabular}

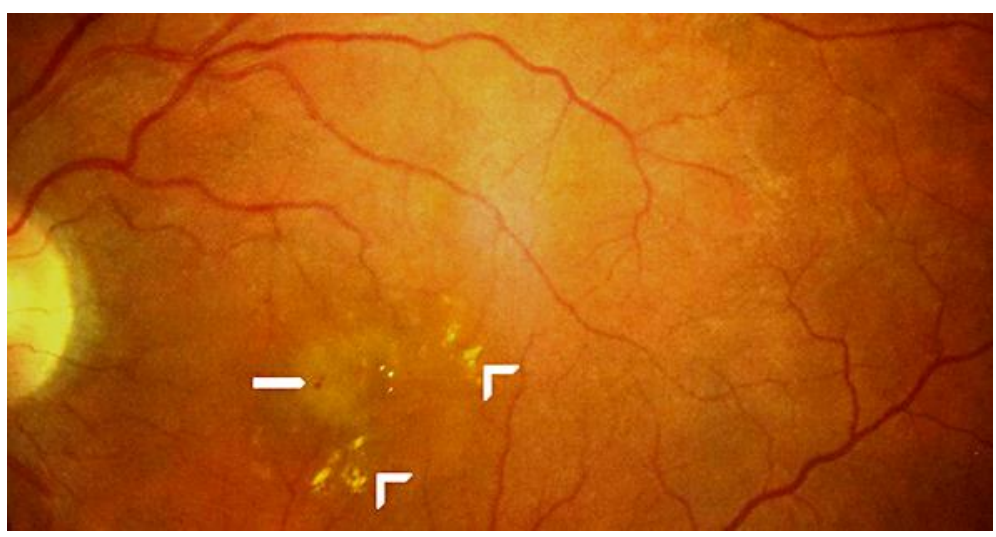

Fig. 1. Color fundus photograph of the LE in an 85-year-old patient with IMT-1 associated with type 3 neovascularization, presenting 2 small hemorrhages nasally to the fovea (arrow), accompanied by lipid exudates (open arrowheads). 


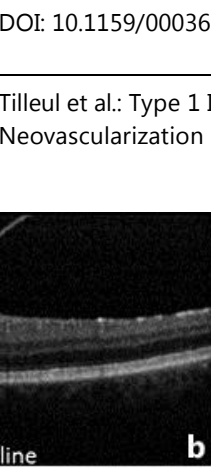

(c) 2014 S. Karger AG, Basel

www.karger.com/cop

Tilleul et al.: Type 1 Idiopathic Macular Telangiectasia Associated with Type 3 Neovascularization
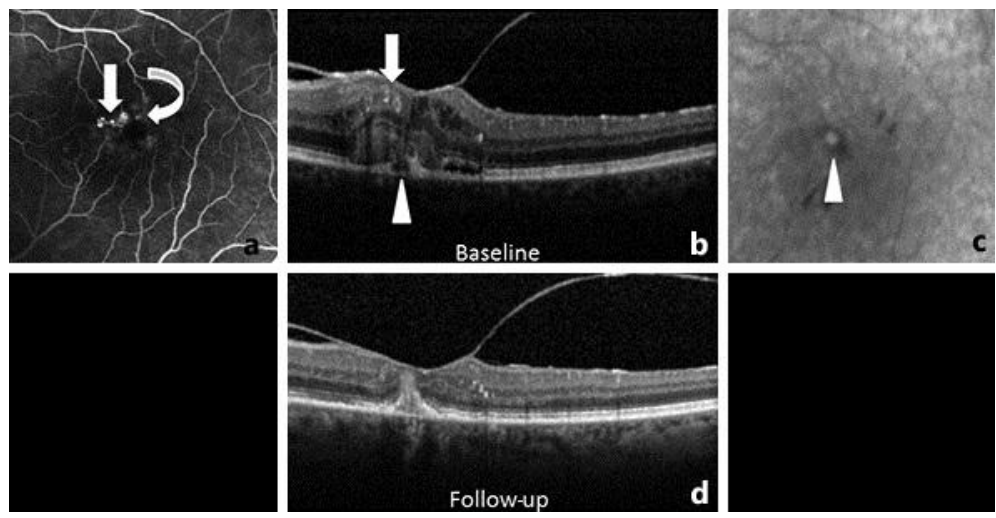

Fig. 2. Multimodal imaging in an 85-year-old patient showing IMT-1 associated with type 3 neovascularization. a Early-phase FA at baseline showing hyperfluorescent lesions nasally to the fovea, corresponding to the dilated capillaries (arrow), and a zone of focal hyperfluorescence with a superotemporal right-angle dilated feeding vessel, deepening into the foveal avascular zone (curved arrow), evocative of a type 3 intraretinal neovascularization. b SD-OCT at baseline showing small, round, well-demarcated foveal lesions with hyperreflective edges, corresponding to microaneurysms, along with cystoid spaces (arrow). A hyperreflective lesion located in the outer retinal layers, adherent to the underlying retinal pigment epithelium, indicates type 3 intraretinal neovascularization (arrowhead). c Late-phase ICGA at baseline showing a focal hyperfluorescence (arrowhead), corresponding to the intraretinal complex of type 3 neovascularization. $\mathbf{d}$ Six-month follow-up SD-OCT revealing the disappearance of both the cystoid macular edema and serous retinal detachment. 